\title{
Impactos sociais na vida da pessoa com implante coclear: uma revisão sistemática
}

Social impacts in the life of person with cochlear implant: a systematic review Impactos sociales en la vida de la persona con implante coclear: una revisión sistemática

\section{Josana Carla Gomes Soares Silva}

Mestranda na Universidade Federal de São Carlos, São Carlos, São Paulo, Brasil. josanacarla.silva@hotmail.com

ORCID - https://orcid.org/0000-0002-3388-5269

\section{Vanessa Cristina Paulino}

Doutora pela Universidade Federal de São Carlos, São Carlos, São Paulo, Brasil.

vanessakju@gmail.com

ORCID - https://orcid.org/0000-0003-0063-0355

\section{Maria da Piedade Resende da Costa}

Professora doutora na Universidade Federal de São Carlos, São Carlos, São Paulo, Brasil. mariadapiedadecostac@gmail.com

ORCID - https://orcid.org/0000-0002-7420-5602

Recebido em 5 de maio 2019

Aprovado em 15 de janeiro de 2020

Publicado em 9 de março de 2020

\section{RESUMO}

Existem divergências quanto ao uso do Implante Coclear (IC); a comunidade surda acredita que seja um retrocesso na luta pelo reconhecimento de sua língua e cultura e a medicina acredita que este seja o modo mais adequado em tratar a deficiência auditiva. Por esses dissensos e considerando a crescente quantidade de cirurgias para IC, se objetivou averiguar a produção nacional acerca dos efeitos sociais advindos do IC na vida do indivíduo implantado. A pesquisa empregou a metodologia da Revisão Sistemática, que buscou revisar bibliografias e delinear um trabalho reflexivo e compreensivo, assim como fazer uma análise histórico-crítica interdisciplinar do tema. A partir dos critérios de inclusão e exclusão determinados, dos 1495 estudos identificados, apenas cinco foram incluídos na revisão. A análise destes permitiu considerar que os efeitos sociais na vida da pessoa implantada tendem a ser negativos quando relacionados ao aspecto físico do dispositivo externo, podendo resultar em vergonha, sentimento de inferioridade e insegurança. Resultou que pode haver rejeição da pessoa implantada pelo grupo ouvinte, devido à dificuldade em falar. Apresentou ainda que os efeitos do IC são distintos em adolescentes e adultos em decorrência, provavelmente, das mudanças enfrentadas nesta etapa de desenvolvimento. Em contrapartida, pessoas que fizeram o IC em decorrência de surdez pós-lingual demonstraram efeitos sociais positivos. Por esses achados e devido à escassez de investigações sobre a temática, se acredita serem primordiais novas 
http://dx.doi.org/10.5902/1984686X38002

pesquisas sobre os efeitos sociais do IC, com o propósito de aprimorar a compreensão de como eles afetam a qualidade de vida da pessoa implantada.

Palavras-chave: Educação Especial; Pessoa surda; Implante Coclear.

\section{ABSTRACT}

There is divergence regarding the use of Cochlear Implant $(\mathrm{Cl})$, between the deaf community and the medicine community. Considering the growing number of surgeries for $\mathrm{Cl}$, the objective was to investigate the national production about the social effects of $\mathrm{Cl}$ on the implanted individual's life. The research employed the methodology of the Systematic Review, which sought to review bibliographies and delineate a reflective and comprehensive work, as well as make an interdisciplinary historical-critical analysis of the theme. From the inclusion and exclusion criteria determined, of the 1495 studies identified, only five were included in the review. The analysis allowed us to consider that the social effects on the implanted person's life tend to be negative when related to the physical aspect of the external device, which may result in shame, feeling of inferiority and insecurity. It was found that there may be rejection of the person implanted by the listener group, due to the difficulty in speaking. It was also identified that the effects of $\mathrm{Cl}$ are distinct in adolescents and adults, probably due to the changes faced at this stage of development. In contrast, people who did $\mathrm{Cl}$ due to post lingual deafness showed positive social effects. Due to these findings and due to the scarcity of research on the subject, it is agreed that new research on the social effects of $\mathrm{Cl}$ is essential, with the purpose of improving the understanding of how they affect the implanted person's quality of life.

Keywords: Special Education; Deaf person; Cochlear Implant.

\section{RESUMEN}

Existen diferencias cuanto al uso del Implante Coclear (IC) entre la comunidad sorda y la comunidad médica. Debido a estas divergencias y considerando una gran cantidad de cirugías para IC, el objetivo de ese estudio fue investigar en la producción nacional los efectos sociales proveniente del IC en la vida del individuo implantado. Se empleó la metodología de revisión sistemática, que buscó revisar bibliografías y delinear un trabajo reflexivo y comprensible, así como un análisis histórico-crítico interdisciplinario del tema. Desde los criterios de inclusión y exclusión determinados, de los 1495 estudios identificados, solo cinco fueron inclusos en la revisión. Además, es posible considerar que los efectos sociales de la vida implantada tienden a ser negativos cuando se relacionan con el aspecto físico del dispositivo externo, lo que puede provocar vergüenza, sensación de inferioridad e inseguridad. Fue averiguado que la persona con IC puede sufrir rechazo por el grupo oyente debido a la dificultad para hablar. Se observa que los efectos del IC son diferentes en adolescentes y adultos, probablemente por la fase del desarrollo. Por el contrario, las personas que hicieron al IC debido a la perdida de la audición mostraron efectos sociales positivos. Por estas razones y debido a la falta de investigación sobre el tema, se acuerda que la investigación sobre los efectos sociales de la IC se hace necesaria para mejorar la comprensión de cómo se ve afectada la calidad de vida del individuo implantado.

Palabras clave: Educación Especial; Persona sorda; Implante Coclear. 
http://dx.doi.org/10.5902/1984686X38002

\section{Introdução}

A medicina utiliza o transplante como meio de substituir um órgão que apresenta mau funcionamento por outro saudável, contudo, tal procedimento apresenta riscos como rejeições, infecções e falta de doadores. Os avanços tecnológicos possibilitaram que a medicina moderna, em conjunto com a engenharia, pudesse contornar esses riscos substituindo órgãos que até pouco tempo não tinham como ser transplantados por meios artificiais (CARVALHO, 1999). No caso da deficiência auditiva, quando a cóclea apresenta mau funcionamento, é indicado o implante coclear (IC), também conhecido como "ouvido biônico" (CARVALHO, 1999).

O IC consiste em um circuito eletrônico com componentes internos e externos que transforma o som ambiental em impulsos elétricos, levando-os à cóclea por um ou mais eletrodos (CARVALHO, 1999). É possível dizer, portanto, que este "ouvido biônico" insere a pessoa surda no mundo sonoro por estimulação elétrica.

Sobre o IC, Lichtiget al. (2003) afirmam que:

De maneira diferente da prótese auditiva, o IC não objetiva amplificar o som, mas sim, melhorar o desempenho e a percepção auditiva. É indicado para pacientes com perda neurossensorial severa ou profunda bilateral, sem contraindicações médicas, com história de consistente uso de prótese auditiva e com plateau ${ }^{1}$ no desenvolvimento da fala e da linguagem. Ainda, os candidatos ao IC devem apresentar atitude positiva com relação ao som, boa possibilidade de gerenciamento familiar e profissional e frequentar ambientes que estimulem a comunicação oral (LICHTIGet al., 2003, p. 03).

Como o implante coclear é inserido por meio cirúrgico, este processo envolve riscos referentes a qualquer cirurgia, como: a) infecções e/ou necrose da pele; b) complicações anestésicas ou pós-operatórias; c) insucesso na colocação do implante, caso ocorram alterações anatômicas no ouvido do paciente; d) paralisia cerebral; e) tontura em decorrência da perfuração da cóclea (órgão do equilíbrio e da audição); f) meningite e fístula liquórica - embora raros atualmente, podem ocorrer no início do implante; g) perda de resquício auditivo por conta da perfuração do sistema auditivo para implantação dos eletrodos; h) não funcionamento do aparelho implantado; e i) deslocamento do eletrodo (IMPLANTE COCLEAR, 2005).

Ressalta-se que, devido ao avanço da medicina, muitos destes riscos, como migração do eletrodo e interrupção de seu funcionamento, podem ser facilmente detectados e tratados (TEFILI, 2013). 
http://dx.doi.org/10.5902/1984686X38002

As autoras Tsukamoto e Fialho (2014) afirmam que o IC pode ser para o indivíduo "[...] um meio facilitador para se atingir a emancipação" (p. 4), aquisição de fluência da língua portuguesa, melhor desempenho auditivo, trazendo ainda a possibilidade de utilização de telefones celulares e a retomada da vida social e profissional (TSUKAMOTO; FIALHO, 2014).

Quanto ao uso de aparelhos auditivos e implante, as autoras Tsukamoto e Fialho (2014), relatam que:

Há pontos divergentes em relação ao uso dos dispositivos eletrônicos para audição, tais como o uso [de] Aparelho Amplificador Sonoro Individual (AASI), o Implante Coclear e o Sistema FM, pois, para o povo surdo estes dispositivos valorizam a hegemonia dos ouvintes sobre os surdos e [...] deturpa a luta surda em relação ao bilinguismo (TSUKAMOTO; FIALHO, 2014, p. 6 - 7).

Apesar dos benefícios versados anteriormente, ainda existem muitas divergências quanto ao implante coclear. A comunidade surda é contrária ao implante por acreditar que este seja um retrocesso na luta pelo reconhecimento da Língua de Sinais, de sua cultura e modo de ser diferente dos ouvintes ou até mesmo pelo desconhecimento quanto aos procedimentos e benefícios do IC. A comunidade médica, por outro lado, o defende como o meio mais assertivo no tratamento de pessoas com deficiência auditiva para que estas possam retomar suas práticas sociais e profissionais, de modo a otimizar suas interações com pessoas ouvintes e serem mais independentes.

Assim, dada a problemática de haver uma quantidade crescente de cirurgias para a implantação coclear, o presente estudo busca dar relevo aos efeitos sociais do IC na vida da pessoa implantada a partir de seus próprios relatos. Para atingir tal objetivo, analisamos de modo sistemático os trabalhos acadêmicos relacionados ao implante coclear em âmbito nacional.

\section{Procedimentos Metodológicos}

Foi aplicada a metodologia da Revisão Sistemática que, de acordo com Cordeiro et al. (2007), se configura como um processo de reunião, avaliação crítica e sintética de resultados de múltiplos estudos pelo qual oportuniza esclarecer questões de pesquisa e qualidade da produção acadêmica em determinada área, podendo indicar problemas metodológicos e pontos ainda não estudados (CORDEIRO et al., 2007).

Fernández-Ríos e Buela-Casal (2009) afirmam que a revisão sistemática é um trabalho reflexivo e compreensivo no qual se faz uma análise histórico-crítica 
http://dx.doi.org/10.5902/1984686X38002

interdisciplinar de um tema, sendo assim muito diferente de um trabalho pautado na simples relação cronológica de exposição linear e descritiva sobre uma temática (FERNANDEZ-RIOS; BUELA-CASAL, 2009).

De acordo com Costa e Zoltowski (2014, p. 56), a "[...] revisão sistemática é um método que permite maximizar o potencial de uma busca, encontrando o maior número possível de resultados de uma maneira organizada". Além disso, este método possibilita que o pesquisador se depare com estudos que discordem de suas hipóteses iniciais, evitando, assim, que a pesquisa fique enviesada. Isto porque pelos procedimentos tradicionais é comum que o indivíduo que faz a busca tenha a tendência de selecionar estudos que corroboram com suas hipóteses iniciais e ignorar os que apontam outras perspectivas (COSTA; ZOLTOWSKI, 2014).

Com base em estudos de Akobeng (2005), o presente trabalho seguiu as seguintes etapas metodológicas: 1) delimitação da questão a ser pesquisada; 2) escolha das fontes de dados; 3) eleição das palavras-chave para a busca; 4) busca e armazenamento dos resultados; 5) seleção de artigos, monografias, dissertações e teses pelo resumo - de acordo com critérios de inclusão e exclusão; 6) extração dos dados dos estudos selecionados; 7) avaliação dos estudos; e 8) síntese e interpretação dos dados. Assim, se buscou averiguar pesquisas que abordem o tema "implante coclear" e "efeitos sociais na vida da pessoa implantada".

As buscas de dados foram realizadas em bases de dados eletrônicas por estas possuírem um acervo abrangente e de fácil acesso, possibilitando atingir um grande número de estudos em um curto período (COSTA; ZOLTOWSKI, 2014). Os bancos utilizados foram: a) Banco online de periódicos da Coordenadoria de Aperfeiçoamento de Pessoal de Nível Superior (CAPES); b) Google Acadêmico; c) Literatura Latino-Americana e do Caribe em Ciências da Saúde (LILACS); e d) Scientific Electronic Library Online (SciELO).

Para as buscas foram utilizados descritores do banco de terminologias da Biblioteca Virtual em Saúde (BVS-Psi), sendo eles "efeitos sociais" e "implante coclear", obtendo retorno apenas ao empregar o segundo termo. A pesquisa delimitou a busca do ano de 1999 ao final de 2017, com o último acesso em maio de 2018, devido ao fato dos estudos encontrados na primeira busca remontarem aos anos de 1999 a 2016 . Ainda, a busca se limitou à produção científica nacional, uma vez que foram encontrados estudos em Língua Portuguesa que tratavam de outros países que não o Brasil. 
http://dx.doi.org/10.5902/1984686X38002

Para delimitação dos trabalhos a serem analisados, foram adotados os seguintes critérios de inclusão: a) ser um artigo científico, monografia, dissertação ou tese; b) apresentar as expressões de busca no título ou resumo; c) abordar os efeitos sociais do implante coclear na vida da pessoa implantada; e d) ter pessoas com implante coclear como respondentes.

Quanto aos critérios de exclusão, foram adotados os seguintes: a) estudos de revisão, observação e ensaios; b) população não-implantada como respondente; c) estudos não encontrados para a leitura na íntegra; d) estudos repetidos; e) estudos com informações insuficientes; e f) estudos que não atendessem aos critérios de inclusão.

Para a análise de dados, foi adaptado o "Roteiro de Análise de Teses e Dissertações" de Nunes, Ferreira e Mendes (2002), do qual foram considerados os seguintes dados referentes a identificação e análise descritiva dos estudos: a) instituição; b) autor e título; c) objetivo; d) método/participantes; e) aspectos avaliados; e f) resultados e conclusão.

A seguir apresentamos os resultados obtidos a partir da aplicação da metodologia exposta.

\section{Resultados}

Por meio das buscas realizadas nas bases de dados eletrônicas, utilizando o descritor "implante coclear", os resultados obtidos foram: a) banco online de periódicos da Coordenadoria de Aperfeiçoamento de Pessoal de Nível Superior (CAPES) = 95 estudos; b) Scientific Electronic Library Online (SciELO) = 160 estudos; c) Literatura LatinoAmericana e do Caribe em Ciências da Saúde (LILACS) = 355 estudos; e d) Google Acadêmico = 885 estudos; totalizando 1495 estudos com o descritor "implante coclear" no título ou resumo.

Foram analisados os títulos e resumos dos 1495 estudos durante a pré-seleção; destes, foram excluídos 1441, pois não atendiam aos critérios de inclusão apresentados anteriormente. Os trabalhos excluídos analisavam a função auditiva, a fala, tipo de dispositivo utilizado para implante coclear, opiniões de profissionais baseadas em experiência clínica, experiências clínicas relacionadas a contextos de outros países e/ou ainda empregavam a metodologia de observação.

Posteriormente, foi feita a leitura na íntegra de 54 estudos; destes, foram excluídos 49, sendo que 14 eram repetidos, tendo sido encontrados em outras bases de dados; 19 
http://dx.doi.org/10.5902/1984686X38002

tinham temas não relacionados aos efeitos sociais decorrentes do implante coclear (como educação, capacidade auditiva, fala, personalidade, dispositivos utilizados para implantação); oito (8) estudos foram excluídos devido ao tipo de metodologia empregada (revisão, ensaio ou observação); sete (7) por não terem pessoas implantadas como respondentes (sendo pais e/ou profissionais dando suas opiniões sobre o implante coclear); e um (1) estudo foi excluído por ter os resultados fora do tema pesquisado, como sistematizado na Figura 1.

Com isso, dos 54 estudos selecionados para leitura integral, apenas cinco (5) foram considerados para a presente revisão sistemática. Um número ainda pequeno, porém significativo, pois retrata que a temática não tem sido muito explorada em âmbito nacional.

Figura 1: Etapas de seleção de estudos para inclusão na revisão sistemática e os motivos de exclusão.

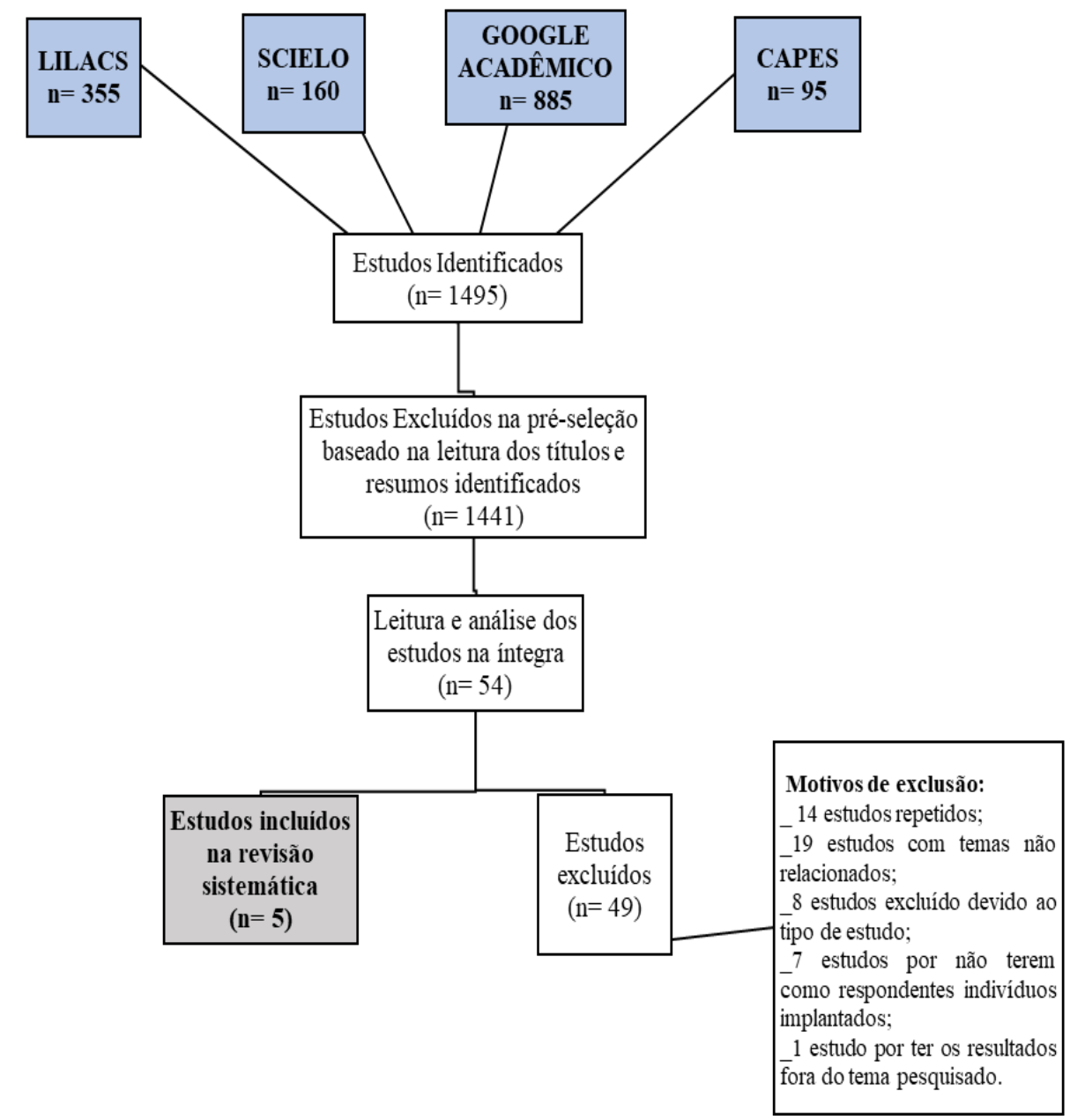

Fonte: Dados da pesquisa. Elaboração própria, 2019.

A partir dos artigos selecionados para revisão, como mostra a Figura 1, foram feitas as análises e sínteses dos dados, conforme mencionado anteriormente, seguindo a 
http://dx.doi.org/10.5902/1984686X38002

adaptação do "Roteiro de Análise de Teses e Dissertações" de Nunes, Ferreira e Mendes (2002). O roteiro foi utilizado com a finalidade de auxiliar a avaliar os estudos localizados, dando direção quanto aos tópicos relevantes a serem analisados e discutidos de modo científico.

A fim de facilitar a análise e organizar os dados, foram construídos quadros sequenciados com as informações retiradas dos estudos, de acordo com a ordem cronológica dos mesmos, indicados nos Quadros 1, 2, 3, 4 e 5.

Quadro 1: Identificação e análise descritiva do estudo de Murakami et al. (2001).

\begin{tabular}{|l|l|}
\hline $\begin{array}{l}\text { Aspectos } \\
\text { analisados }\end{array}$ & Dados coletados \\
\hline Instituição & $\begin{array}{l}\text { Programa de Implante Coclear do Centro de Pesquisas Audiológicas do Hospital } \\
\text { de Reabilitação de Anomalias Craniofaciais da Universidade de São Paulo (USP) } \\
\text { - Campus de Bauru. }\end{array}$ \\
\hline Autor e título & $\begin{array}{l}\text { MURAKAMI, G. A. O. et al. Expectativas prévias ao implante coclear e avaliação } \\
\text { pós-implante em adolescentes. }\end{array}$ \\
\hline Objetivo & $\begin{array}{l}\text { Verificar tendências de relação entre expectativas prévias (EP), ansiedades e } \\
\text { temores de pacientes candidatos a implante coclear (IC) e suas avaliações } \\
\text { posteriores quanto a ganhos obtidos com o implante. }\end{array}$ \\
\hline Método/Participantes & $\begin{array}{l}\text { Estudo: Qualitativo e quantitativo } \\
\text {-Participantes: } 6\end{array}$ \\
& $\begin{array}{l}\text {-Privação auditiva: } 4 \text { a 10 anos } \\
\text {-Tempo de utilização do IC: } 7 \text { a } 44 \text { meses } \\
\text { Idade dos participantes: } 13 \text { a 19 anos } \\
\text {-Grupo controle: não }\end{array}$ \\
\hline Aspectos avaliados & $\begin{array}{l}\text { Dificuldades enfrentadas por tais pacientes diante da indicação do IC em relação } \\
\text { às suas expectativas, temores, ansiedades e conflitos diante da possibilidade de } \\
\text { ouvir melhor e das demandas de readaptação de sua autoimagem e } \\
\text { autoconceito. }\end{array}$ \\
\hline Resultados/ \\
Conclusão & $\begin{array}{l}\text { Antes da cirurgia de IC todos os participantes relataram desagrado e } \\
\text { preocupação com a estética do aparelho, assim como tinham altas expectativas } \\
\text { quanto a voltar a ouvir. Após o implante os pacientes afirmaram sua satisfação } \\
\text { argumentando que o aparelho ajudava muito na audição, que suas vidas se } \\
\text { transformaram para melhor e que estavam muito felizes; contudo, alguns } \\
\text { relataram continuar a sentir vergonha e não gostar do aparelho e de sua estética. } \\
\text { Outros pacientes informaram que precisaram mudar suas rotinas após o } \\
\text { implante. Quanto à correspondência destes resultados com as expectativas } \\
\text { prévias ao implante, uma metade relata que pensou que seria melhor, a outra } \\
\text { que o resultado foi melhor do que imaginavam. }\end{array}$ \\
\hline
\end{tabular}

Fonte: Dados da pesquisa. Elaboração própria, 2019. 
http://dx.doi.org/10.5902/1984686X38002

Quadro 2: Identificação e análise descritiva do estudo de Sant'Anna, Eichner e Guedes

\begin{tabular}{|l|l|}
\hline Aspectos analisados & Dados coletados \\
\hline Instituição & Centro de Estudo e Reabilitação "CER - Fonoaudiologia". \\
\hline Autor e título & \multicolumn{1}{|c|}{ SANT'ANNA, S. B. G.; EICHNER, A. C. O.; GUEDES, M. C. Benefício do } \\
implante \\
coclear em indivíduos adultos com surdez pré-lingual.
\end{tabular}

Fonte: Dados da pesquisa. Elaboração própria, 2019.

Quadro 3: Identificação e análise descritiva do estudo de Zanardi, Yamada e Bevilacqua (2009)

\begin{tabular}{|l|l|}
\hline $\begin{array}{l}\text { Aspectos } \\
\text { analisados }\end{array}$ & Dados coletados \\
\hline Instituição & $\begin{array}{l}\text { Centro de Pesquisas Audiológicas do Hospital de Reabilitação de Anomalias } \\
\text { Craniofaciais da Universidade de São Paulo (USP) - Campus de Bauru. }\end{array}$ \\
\hline Autor e título & $\begin{array}{l}\text { ZANARDI, M. B.; YAMADA, M. O.; BEVILACQUA, M. C. A experiência do adolescente } \\
\text { usuário de implante coclear. }\end{array}$ \\
\hline Objetivo & $\begin{array}{l}\text { Compreender a vida do adolescente com IC dentro de uma perspectiva fenomenológica } \\
\text { e delinear como o fenômeno da adolescência é vivido nos diversos contextos e } \\
\text { aspectos: escola, família, amizade, namoro, enfim, questões que permeiam o fenômeno } \\
\text { adolescer. }\end{array}$ \\
\hline Método/ & $\begin{array}{l}\text {-Estudo: fenomenológico } \\
\text { Participantes } \\
\text {-Privação auditiva: } 13 \text { a } 16 \text { anos } \\
\text {-Tempo de utilização do IC: } 10 \text { a } 12 \text { anos } \\
\text {-Idade dos participantes: } 13 \text { a } 16 \text { anos } \\
\text {-Grupo controle: não }\end{array}$ \\
\hline
\end{tabular}


Quadro 3: Identificação e análise descritiva do estudo de Zanardi, Yamada e Bevilacqua (2009)

\begin{tabular}{|l|l|}
\hline $\begin{array}{l}\text { Aspectos } \\
\text { analisados }\end{array}$ & Dados coletados \\
\hline $\begin{array}{l}\text { Aspectos } \\
\text { avaliados }\end{array}$ & $\begin{array}{l}\text { Como o fenômeno da adolescência é vivido nos diversos contextos e aspectos: escola, } \\
\text { família, amizade, namoro, enfim, questões que permeiam o fenômeno adolescer. }\end{array}$ \\
\hline $\begin{array}{l}\text { Resultados/ } \\
\text { Conclusão }\end{array}$ & $\begin{array}{l}\text { O estudo feito após o implante coclear concluiu que o contexto escolar era marcado por } \\
\text { dificuldade e constrangimento em não compreender bem a língua portuguesa. O contexto } \\
\text { familiar foi tido como um espaço de apoio. O relacionamento afetivo era marcado pelos } \\
\text { sentimentos de vergonha, inferioridade, preconceito e estereótipo. Quanto à amizade, o IC } \\
\text { favoreceu a comunicação e o relacionamento interpessoal, mesmo tendo a exclusão e } \\
\text { rejeição como parte da realidade escolar, assim como o estigma da deficiência auditiva. O } \\
\text { IC, para os participantes, significava satisfação (se referiram à importância de ouvir a voz } \\
\text { das pessoas, de falar ao telefone, de acompanhar melhor os estudos) e, por outro lado, } \\
\text { significava a constatação da deficiência auditiva e da dificuldade de aceitação. }\end{array}$ \\
\hline
\end{tabular}

Fonte: Dados da pesquisa. Elaboração própria, 2019.

Quadro 4: Identificação e análise descritiva do estudo de Yamada e Bevilacqua (2012)

\begin{tabular}{|l|l|}
\hline $\begin{array}{l}\text { Aspectos } \\
\text { analisados }\end{array}$ & Dados coletados \\
\hline Instituição & $\begin{array}{l}\text { Centro de Pesquisas Audiológicas do Hospital de Reabilitação de Anomalias } \\
\text { Craniofaciais da Universidade de São Paulo (USP) - Campus de Bauru. }\end{array}$ \\
\hline Autor e título & $\begin{array}{l}\text { YAMADA, M. O.; BEVILACQUA, M. C. Dimensão afetiva da pessoa com surdez } \\
\text { adquirida, antes e após o implante coclear. }\end{array}$ \\
\hline Objetivo & $\begin{array}{l}\text { Averiguar, antes e após o uso do IC, a dimensão afetiva em pacientes com } \\
\text { surdez adquirida e investigar as modalidades dos sentimentos egoicos, } \\
\text { sentimentos em relação ao próximo, sentimentos de temporalidade estados de } \\
\text { ânimo ou humor. }\end{array}$ \\
\hline Método/Participantes & $\begin{array}{l}\text {-Estudo: fenomenológico } \\
\text {-Participantes: 44 } \\
\text { _Privação auditiva: não especificado } \\
\text {-Tempo de utilização do IC: não especificado } \\
\text { _ldade dos participantes: } 21 \text { a } 68 \text { anos } \\
\text { _Grupo controle: não }\end{array}$ \\
\hline Aspectos avaliados & $\begin{array}{l}\text { Se deu enfoque aos sentimentos e aos estados de ânimo da pessoa com surdez } \\
\text { adquirida e usuária do IC. }\end{array}$ \\
\hline $\begin{array}{l}\text { Resultados/ } \\
\text { Conclusão }\end{array}$ & $\begin{array}{l}\text { Concluiu que, na vivência da surdez, houve predomínio de sentimentos negativos } \\
\text { e de um clima afetivo de tensão e depressão que levava o sujeito a uma } \\
\text { vinculação negativa, de assintonia com o mundo. Entretanto, na vivência com o } \\
\text { IC, houve predomínio de sentimentos positivos e de um clima afetivo de } \\
\text { tranquilidade e contentamento, observando assim, uma vinculação positiva do } \\
\text { sujeito e sintonia com o mundo. }\end{array}$ \\
\hline
\end{tabular}

Fonte: Dados da pesquisa. Elaboração própria, 2019. 
http://dx.doi.org/10.5902/1984686X38002

Quadro 5: Identificação e análise descritiva do estudo de Buarque, et al. (2014)

\begin{tabular}{|l|l|}
\hline $\begin{array}{l}\text { Aspectos } \\
\text { analisados }\end{array}$ & Dados coletados \\
\hline Instituição & Programa de Implante Coclear do Otocentro-RN, Natal, RN. \\
\hline Autor e título & $\begin{array}{l}\text { BUARQUE, L. F. S. F. P. et al.. Satisfação dos usuários do implante coclear com } \\
\text { perda auditiva pós-lingual. }\end{array}$ \\
\hline Objetivo & Avaliar a satisfação dos usuários de IC com perda auditiva pós-lingual. \\
\hline Método/ & $\begin{array}{l}\text {-Estudo: seccional } \\
\text { Participantes } \\
\text { _Privação auditiva: } 17 \text { anos em média } \\
\text {-Tempo de utilização do IC: } 21 \text { meses em média } \\
\text { _Idade dos participantes: } 46 \text { anos em média } \\
\text { _Grupo controle: sim }\end{array}$ \\
\hline Aspectos avaliados & $\begin{array}{l}\text { Questões fundamentais que envolvem as expectativas do usuário, dimensões } \\
\text { psicológicas, dificuldades que ainda permanecem mesmo com o uso do } \\
\text { dispositivo, problemas no processo de reabilitação, dentre outros. }\end{array}$ \\
\hline $\begin{array}{l}\text { Resultados/ } \\
\text { Conclusão }\end{array}$ & $\begin{array}{l}\text { Baixo índice, porém expressivo, de indivíduos insatisfeitos. De modo geral, os } \\
\text { usuários de IC possuem um alto nível de satisfação. Os fatores mais evidentes de } \\
\text { insatisfação se relacionam com os serviços e custos com o IC, imagem pessoal e } \\
\text { fatores negativos com o uso do dispositivo, como por exemplo, ouvir em ambientes } \\
\text { ruidosos. }\end{array}$ \\
\hline
\end{tabular}

Fonte: Dados de pesquisa. Elaboração própria, 2019.

Nos Quadros 1, 2, 3, 4 e 5 são apresentadas informações retiradas dos estudos incluídos nesta revisão sistemática, a qual trata dos efeitos sociais advindos do implante coclear na vida da pessoa implantada. A seguir, será apresentada a análise e discussão concernente aos resultados levantados a partir da pesquisa.

\section{Análise e discussão dos dados}

Todos os estudos selecionados são originais em formato de artigo científico. Quatro (4) deles foram realizados no estado de São Paulo, na região onde se encontram Centros que realizam o procedimento de implante coclear, nas cidades de Bauru e Ribeirão Preto. Um (1) estudo aconteceu em Natal, no estado de Rio Grande do Norte. Estes dados evidenciam uma maior produção científica acerca dos efeitos do implante coclear na região Sudeste do país. 
http://dx.doi.org/10.5902/1984686X38002

Sobre o tema, todos os artigos tratam dos efeitos sociais do IC na vida da pessoa implantada, abordando ainda fenômenos relativos à expectativa, benefício, adolescência, dimensão afetiva e satisfação do usuário de IC. Apesar de os trabalhos analisados apresentarem objetivos distintos, estes tinham em comum a intenção de estudar os fenômenos e efeitos subjetivos advindos do implante coclear.

Murakamiet al. (2001) trataram de verificar tendências das relações entre expectativas prévias, ansiedade e temores dos candidatos quanto ao IC, com suas avaliações posteriores. Tendo seus objetivos alcançados, evidenciou também aspectos negativos acerca do implante.

O trabalho de Sant'Anna, Eichner e Guedes (2008) avaliou os benefícios obtidos por jovens adultos com surdez pré-lingual após a colocação do implante coclear. Considerou, além dos testes de percepção de fala, também as impressões subjetivas dos participantes em relação a mudanças na qualidade de vida destes. Os objetivos foram alcançados no trabalho.

A pesquisa de Zanardi, Yamada e Bevilacqua (2009) buscou compreender a vida do adolescente com implante coclear dentro da perspectiva fenomenológica, que consiste em estudar a essência das coisas e como estas são percebidas no mundo, e delinear como o fenômeno da adolescência é vivido nos diversos contextos e aspectos sociais. Para tal, foram analisados o contexto escolar, de amizades, namoro e outras questões que permeiam o fenômeno adolescer, tendo atingido esses objetivos.

O trabalho de Yamada e Bevilacqua (2012) se empenhou em averiguar, antes e após o uso de IC, a dimensão afetiva em pacientes com surdez adquirida e investigar as modalidades dos sentimentos egóicos (ou seja, sentimentos voltados para o ego, para si), sentimentos em relação ao próximo, sentimentos de temporalidade e estados de ânimo ou humor, tendo alcançado tais objetivos.

A pesquisa de Buarque et al. (2014) avaliou a satisfação dos usuários de implante coclear com perda auditiva pós-lingual, tendo atingido seus objetivos.

Quanto ao Método/Participantes, dois (2) estudos realizaram pesquisas fenomenológicas; uma (1) clínica retrospectiva de corte transversal; uma (1) seccional; e uma (1) qualitativa e quantitativa.

Com relação aos Participantes, os trabalhos contaram com uma variação de 4 a 51 indivíduos. A idade dos participantes variou entre 13 e 68 anos. A média de privação auditiva entre os participantes dos estudos analisados foi de 15 anos. O uso de implante 
coclear teve a média de 74 meses de utilização. Dos cinco (5) trabalhos selecionados, apenas um (1) usou grupo controle, como é possível observar na Tabela 1.

Tabela 1: Dados dos estudos analisados

\begin{tabular}{|c|c|c|c|c|c|c|}
\hline $\begin{array}{l}\text { Trabalh } \\
\text { o } \\
\text { analisad } \\
\text { o }\end{array}$ & Instituição & $\begin{array}{l}\text { Participante } \\
\text { s }\end{array}$ & $\begin{array}{l}\text { Idade } \\
\text { (anos) }\end{array}$ & $\begin{array}{c}\text { Privação } \\
\text { Auditiva } \\
\text { (anos) }\end{array}$ & $\begin{array}{c}\text { Uso de } \\
\text { IC } \\
\text { (meses) }\end{array}$ & $\begin{array}{c}\text { Grupo } \\
\text { Control } \\
\text { e }\end{array}$ \\
\hline T1 & $\begin{array}{l}\text { Programa de Implante Coclear do } \\
\text { Centro de Pesquisas Audiológicas do } \\
\text { Hospital de Reabilitação de Anomalias } \\
\text { Craniofaciais da Universidade de São } \\
\text { Paulo, Campus de Bauru }\end{array}$ & 6 & $13-19$ & $4-10$ & $7-44$ & Não \\
\hline T2 & $\begin{array}{l}\text { Centro de Estudo e Reabilitação "CER } \\
\text { - Fonoaudiologia" }\end{array}$ & 11 & $16-31$ & $16-31$ & $4-60$ & Não \\
\hline T3 & $\begin{array}{l}\text { Centro de Pesquisas Audiológicas do } \\
\text { Hospital de Reabilitação de Anomalias } \\
\text { Craniofaciais da Universidade de São } \\
\text { Paulo, Campus de Bauru }\end{array}$ & 4 & $13-16$ & $13-16$ & $\begin{array}{c}120- \\
144\end{array}$ & Não \\
\hline T4 & $\begin{array}{l}\text { Centro de Pesquisas Audiológicas do } \\
\text { Hospital de Reabilitação de Anomalias } \\
\text { Craniofaciais da Universidade de São } \\
\text { Paulo, Campus de Bauru }\end{array}$ & 44 & $21-68$ & - & - & Não \\
\hline T5 & $\begin{array}{l}\text { Programa de Implante } \\
\text { Otocentro-RN, Natal, RN }\end{array}$ & 51 & $\begin{array}{c}46^{*} \\
\text { (média) }\end{array}$ & $\begin{array}{c}17^{*} \\
\text { (média) }\end{array}$ & $\begin{array}{c}21^{*} \\
\text { (média) }\end{array}$ & Sim \\
\hline
\end{tabular}

Fonte: Dados das pesquisas. Elaboração própria, 2019.

Legenda: *Os dados estão discriminados de acordo com os estudos originais².

$\mathrm{Na}$ Tabela 1 são apresentados os dados dos estudos analisados. Os trabalhos tiveram variação entre 4 e 51 indivíduos participantes, tempo de privação auditiva entre 4 e 31 anos, sendo que um (1) estudo não discriminou a idade dos participantes. Quanto ao uso do implante coclear, o maior tempo de utilização foi de 144 meses e o menor de 4 meses.

Com relação aos participantes, foi observado que, no estudo de Murakami et al. (2001), colaboraram quatro (4) indivíduos do sexo masculino e dois (2) do sexo feminino; o estudo de Sant'Anna, Eichner, Guedes (2008) teve cinco (5) participantes do sexo masculino e seis (6) do sexo feminino, enquanto o estudo de Zanardi e Bevilacqua (2009) contou com quatro (4) participantes do sexo feminino e nenhum do sexo masculino. Já o estudo de Yamada e Bevilacqua (2012) teve 44 participantes ao todo, porém não está discriminada a quantidade exata de participantes de cada sexo, apenas que eram de 
ambos, e o estudo de Buarque et al. (2014) contou com 29 indivíduos do sexo masculino e 22 do sexo feminino, como indica o Gráfico 1.

Gráfico 1: Participantes dos estudos analisados

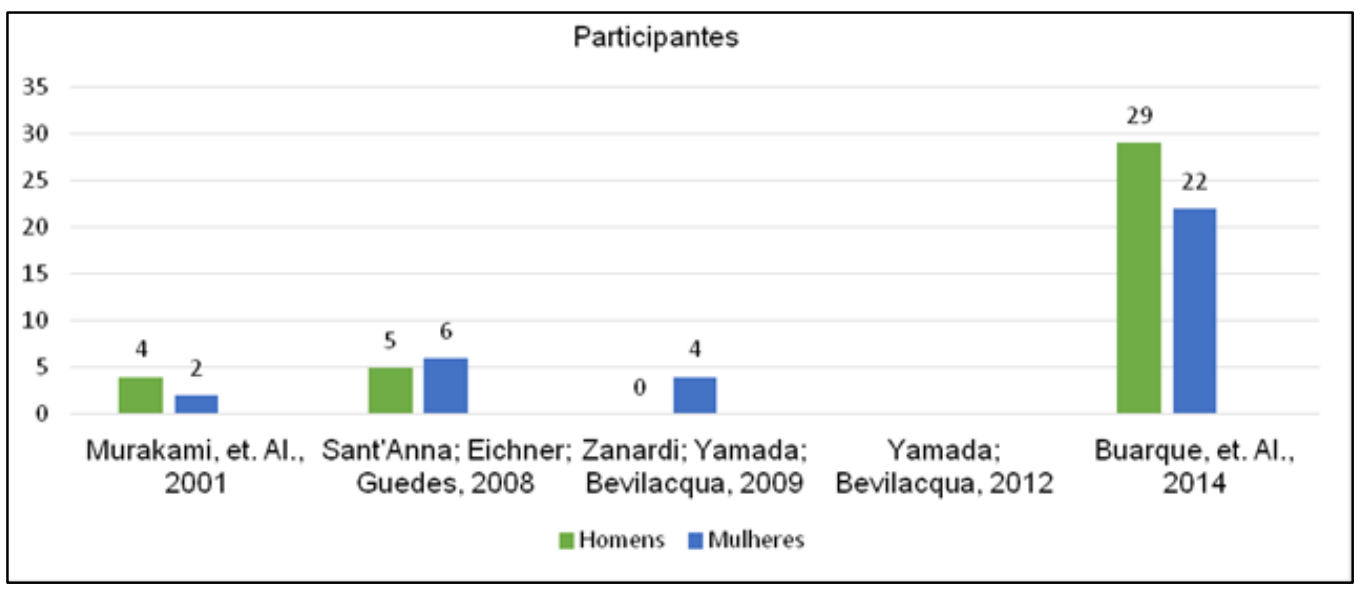

Fonte: Dados da pesquisa. Elaboração própria, 2019.

Considerando os cinco (5) estudos analisados, o total de participantes foi de 116 indivíduos, sendo 38 do sexo masculino e 34 do sexo feminino, não entrando nesta somatória os dados dos participantes do quarto estudo por este não ter especificado a quantidade de participantes de cada sexo.

A partir da análise dos artigos é possível perceber que as queixas sobre o aspecto físico do dispositivo, insatisfação com a autoimagem após o implante, vergonha e sentimentos de inferioridade foram relatados por participantes de ambos os gêneros com idade entre 13 e 21 anos e por mulheres nos estudos com foco em adultos. Os demais participantes não alegaram insatisfação com a aparência do aparelho.

Outro dado relevante é o fato de todos os trabalhos analisados terem como respondentes os próprios usuários do implante coclear, como informado nos critérios de inclusão, o que denota uma maior fidelidade aos resultados.

Os aspectos avaliados pelos estudos foram: a) dificuldades enfrentadas diante da indicação do implante; b) expectativas; c) ansiedade; d) autoimagem; e) percepção da fala; f) qualidade de vida; g) fenômeno da adolescência nos contextos escolar, familiar, amizade e namoro; h) sentimentos; i) estado de ânimo; j) dimensões psicológicas; e k) reabilitação.

Três (3) estudos analisados avaliaram a ansiedade e expectativas quanto ao implante coclear, antes e após o procedimento, outros três (3) analisaram a percepção da fala, dois (2) estudaram os efeitos do implante em adolescentes, sendo que um (1) deles 
http://dx.doi.org/10.5902/1984686X38002

analisou os efeitos em diversos contextos e aspectos como família, amizade, escola e namoro. Cinco (5) pautaram a qualidade de vida após o implante coclear, dois (2) avaliaram as dificuldades enfrentadas diante da indicação do implante, estado de ânimo e temores e um (1) estudo avaliou a autoimagem.

Em relação aos resultados, os estudos de Murakami et al. (2001) e de Zanardi, Yamada, Bevilacqua (2009), trataram dos efeitos sociais na vida de adolescentes com implante coclear, tendo participantes de idades entre 13 e 19 anos. Os resultados encontrados indicaram que estes participantes se preocupavam com a questão estética do aparelho, no que concerne ao dispositivo externo. Antes e após o procedimento de implante, eles indicaram que este "[...] significava a constatação da deficiência auditiva e da dificuldade de aceitação" (ZANARDI; YAMADA; BEVILACQUA, 2009, p. 85), o que os deixava tristes e com vergonha.

Estes indivíduos ainda relataram preconceito e até mesmo estereótipos ligados à pessoa surda. Uma das participantes do estudo realizado por Zanardi, Yamada, Bevilacqua (2009) relatou, por exemplo, que os surdos seriam barulhentos e briguentos. Para as autoras, tais sentimentos são justificados pela "[...] dificuldade de comunicação com pessoas surdas, preconceito e estereótipo que legitimam a diferença e consequente exclusão daquele que tem limitações maiores de audição, fala e comunicação" (ZANARDI; YAMADA; BEVILACQUA, 2009, p. 81). Deste modo, a pesquisa denotou que os participantes sentiram vergonha e constrangimento com o uso do dispositivo externo. Ainda, uma das participantes relatou que foi rejeitada por suas colegas de escola, tendo sido, literalmente, empurrada para longe do grupo de adolescentes, sofrendo queda ao chão, devido a seu modo diferente de falar (ZANARDI; YAMADA; BEVILACQUA, 2009).

Em contrapartida, no estudo de Murakami et al. (2001), os participantes relataram mudança de suas vidas para melhor após o implante: metade dos participantes relatou não sentir vergonha quanto ao dispositivo externo, estavam satisfeitos com o resultado. Dois deles, porém, disseram ter tido que mudar suas rotinas, não podendo mais praticar esportes radicais, alegando também que o implante dificultou que conseguissem um namoro. Outro participante não gostou de ter feito o implante e relatou que, se tivesse que escolher novamente, não o faria.

Acerca das expectativas quanto ao IC, estes estudos indicaram, de modo geral, um sentimento de satisfação por parte dos participantes. Estes relataram gostar de poder ouvir, mesmo alguns tendo indicado insatisfação e frustração acerca da expectativa que 
http://dx.doi.org/10.5902/1984686X38002

tinham antes do implante de que poderiam falar ao telefone. Foi exposto que tal prática não era possível, pois não ouviam tão bem assim.

Foram descritas também dificuldades de audição na escola, tendo sido mencionado dificuldade em compreender o professor. Foram manifestados, ainda, sentimentos de inferioridade, vergonha e incômodo por ter de pedir ao professor que repetisse suas falas por várias vezes. Outros, no entanto, citaram não se incomodar em pedir para que as pessoas repetissem alguma informação (MURAKAMI et al., 2001, ZANARDI; YAMADA; BEVILACQUA, 2009).

Tais relatos evidenciam que, embora o adolescente com implante coclear se sinta satisfeito com o resultado de poder ouvir e interagir com seus pares, o dispositivo externo pode provocar um efeito negativo nas relações afetivas, como amizade (ZANARDI; YAMADA; BEVILACQUA, 2009).

Outros efeitos sociais advindos do implante coclear relatados pelos indivíduos dos estudos em questão são a mudança de rotina. A vivência emocional dos adolescentes sofreu efeitos advindos do implante tais como ansiedade, mudança da autoimagem e esquema corporal, tendo repercussões em suas dinâmicas pessoais (MURAKAMl et al., 2001). Tais fenômenos não foram estudados nos artigos em questão, apontando para a necessidade de que estes sejam analisados de modo mais acurado a fim de determinar quais fatores podem possibilitar uma melhor adaptação após o implante coclear de modo a facilitar a experiência do adolescente implantado.

No estudo de Sant'Anna, Eichner, Guedes (2008) foi evidenciado que "[...] a avaliação subjetiva de qualidade de vida mostrou que o implante coclear aumenta significativamente a inserção social e profissional do indivíduo" (p. 241). A pesquisa ainda demonstrou que "[...] todos os indivíduos relataram aumento da autoconfiança e melhora do bem-estar geral após o uso do implante coclear" (p. 241). Os efeitos sociais relatados neste estudo foram de uma inserção social e profissional mais adequada, pois com 0 implante coclear os indivíduos passaram a ouvir e compreender os sons, mesmo em ambientes com ruído.

Quanto à qualidade de vida, não fica claro no texto se os dados apresentados são da pesquisa ou de outra fonte consultada. Contudo, os autores afirmam que é necessário haver o acompanhamento a longo prazo dos indivíduos, bem como desenvolver a avalição dos aspectos psicossociais menos específicos, uma vez que o estudo avaliou apenas o reconhecimento de frases e sentenças, e se o IC possibilitava uma boa inserção 
http://dx.doi.org/10.5902/1984686X38002

social no ambiente de trabalho através de questionário (SANT'ANNA; EICHNER; GUEDES, 2008).

O estudo de Yamada e Bevilacqua (2012) avaliou a dimensão afetiva da pessoa usuária de implante coclear antes e após o procedimento e teve como resultado dados que denotaram que antes do implante os indivíduos expressavam sentimentos negativos, como inferioridade, solidão, tristeza, angústia, desânimo, insatisfação, isolamento, desvalor de si mesmos e rejeição. Também apresentavam um clima afetivo de tensão e depressão que levava o sujeito a uma vinculação negativa, de assintonia, com o mundo.

Após o implante coclear foi realizado novo teste e este demonstrou que, na vivência com o implante, predominaram sentimentos positivos como felicidade, valor próprio, autoconfiança, autoaceitação, segurança, contentamento, alegria, cordialidade, satisfação e disposição. Ainda foi observado um clima afetivo de tranquilidade e contentamento a partir da vinculação positiva do sujeito com o mundo (YAMADA; BEVILACQUA, 2012).

Os efeitos sociais advindos do implante coclear, no caso dos participantes deste estudo, foram satisfação, autoconfiança readquirida pela possibilidade de voltar a ouvir, maior participação em atividades sociais, melhora da comunicação no cotidiano e efeitos positivos na afetividade do sujeito, pois passou a existir "[...] a possibilidade de que nestes casos os efeitos sociais sejam mais positivos, que nos casos em que o indivíduo não tenha uma capacidade auditiva satisfatória desde uma idade precoce ou tenha nascido surdo" (SILVA, 2018, p. 08).

Contudo, o estudo de Yamada e Bevilacqua (2012) aborda a surdez como algo negativo em que a pessoa surda/deficiente auditiva esteja sempre deprimida e tensa, sem esperanças para o futuro ou a possibilidade de se comunicar com o próximo e que, havendo a tentativa, esta acaba sendo constrangedora tanto para a pessoa surda quanto para o ouvinte.

O estudo de Buarque et al. (2014), que avaliou a satisfação dos usuários do implante coclear com perda auditiva pós-lingual, demonstrou nos resultados que os participantes estavam satisfeitos com seus dispositivos, obtendo alta pontuação na análise global dos questionários aplicados. Estes relataram que o IC "[...] os auxilia a compreender as pessoas com quem mais conversam como também reduz o número de vezes que solicitam a seus interlocutores que repitam a mensagem falada." (p. 1080). 0 estudo ainda indicou que resultados negativos foram pouco relatados e que estes estavam relacionados, especificamente, à percepção de som em ambientes ruidosos. 
Ainda no estudo de Buarqueet et al. (2014), os participantes demonstraram insatisfação quanto à imagem pessoal após o implante, fato que as autoras sugerem ser devido à aparência do dispositivo. Os sujeitos ainda demonstraram estar "[...] satisfeitos no que diz respeito ao seu dispositivo e o relacionamento no meio social em que vive[m]" (BUARQUE et al., 2014, p. 1085). O estudo relata que a "[...] autoestima e a autonomia são aspectos negativamente afetados na vida da pessoa que não ouve" (p. 1085) e que após o implante coclear estas pessoas relataram sentir mais alegria de viver. Tal dado pode representar um viés na pesquisa já que esta não demonstra dados randomizados justificando tal afirmativa (BUARQUE et al., 2014).

Os efeitos sociais encontrados neste estudo foram a melhora no convívio social em consequência da conquista de autonomia e da melhora significativa da audição. Também foi indicado haver insatisfação quanto à imagem pessoal, o que corrobora com estudos já apresentados (BUARQUE et al., 2014).

Alguns estudos relataram a deficiência auditiva como algo negativo, sem possibilidade de futuro e um estado depressivo, autoagressivo e agressivo, diferente do que apontam alguns teóricos da área, os quais evidenciam que a deficiência auditiva/surdez não interfere na possibilidade de que a pessoa tenha uma vida produtiva, feliz e comunicativa (SACKS, 2010, REZENDE, 2010).

Dentre os estudos analisados foi atentado que apenas um (1) retratou efeitos negativos decorrentes do implante coclear em relação ao custo com a manutenção do dispositivo após o procedimento, dois (2) retratavam efeitos negativos alusivos à autoimagem, um (1) retratou efeitos negativos nas interrelações, um (1) retratou efeitos negativos quanto à mudança de rotina após o implante e um (1) pontuou apenas os efeitos positivos, tendo somente citado que os participantes "reclamaram do implante" (ZANARDI; YAMADA; BEVILACQUA, 2009).

Outros aspectos negativos oriundos do implante coclear observados nos estudos analisados foram: insatisfação com a qualidade de audição (voz robotizada, baixa audição, dificuldade em compreender em ambientes ruidosos); constrangimento em falar e não ser compreendido e por não compreender o interlocutor; dificuldades no relacionamento afetivo resultante da aparência estética do dispositivo externo; a constatação de dificuldade de compreensão e de fala; o IC enquanto afirmação da deficiência auditiva. 
http://dx.doi.org/10.5902/1984686X38002

É importante ressaltar que a qualidade de vida e os aspectos psicossociais após o implante coclear, tal como a vivência emocional dos adolescentes implantados, não foram abordados significativamente nos estudos analisados, assim sendo, estes fenômenos necessitam ser estudados para que haja uma boa adaptação após o procedimento, "[...] de modo a avaliar quais metodologias podem ser aplicadas para que efeitos negativos sejam amenizados e como estes afetam a vida da pessoa implantada" (SILVA, 2018, p. 08).

Foi levantada a hipótese de que os efeitos sociais após o implante coclear são distintos em adolescentes e adultos, pois os estudos realizados com participantes adolescentes tiveram resultados negativos, possivelmente em consequência da autoimagem, mudanças corporais, namoro e interações sociais. Segundo Amaral (2007), ter um corpo em mutação leva a conflitos com a autoimagem, fazendo com que a pessoa sinta vergonha do próprio corpo. Desta maneira é possível que metade dos participantes demonstraram sentir insatisfação, vergonha e tristeza devido ao aspecto físico do dispositivo externo do implante coclear. Este fenômeno foi observado nos estudos analisados e vai ao encontro com as palavras de Martins (1987, p. 28, apud SILVA, 2011, p. 6) ao afirmar que:

A caracterização da adolescência não constitui tarefa muito fácil, porque aos fatores biológicos específicos, atuantes na faixa etária, se somam as determinantes socioculturais, advindas do ambiente onde o fenômeno da adolescência ocorre (MARTINS, 1987, p. 28, apud., SILVA, 2011, p. 6).

Desta maneira, o adolescente que é implantado passa por fenômenos típicos da adolescência acrescidos por estressores que levam a enfrentar situações adversas como a aceitação do grupo diante de sua dificuldade de compreensão e de fala, aparência do dispositivo, curiosidade acerca do mesmo e, ainda, às mudanças de rotina devido ao implante e ao esforço que a reabilitação exige, como é relatado nos estudos de Murakami (2001) e Zanardi, Yamada, Bevilacqua (2009).

De acordo com o levantamento, é possível observar que: a) os indivíduos implantados tinham um histórico de uso de aparelhos auditivos como aparelho de amplificação sonora individual (AASI) e/ou prótese auditiva anterior ao implante coclear; b) muitos participantes apresentaram atitude positiva em relação ao implante; c) tais dados foram levantados nos estudos a partir de questionários respondidos pelos próprios candidatos ao implante; d) alguns participantes apresentaram preocupação quanto ao 
procedimento e ao aspecto físico do IC e quanto à possibilidade de utilizar o telefone celular para conversar.

Foi verificado, ainda, que os participantes fizeram testes de compreensão de fala antes e após o implante coclear, acompanhamento psicológico e triagem com equipe multidisciplinar, bem como acompanhamento após o procedimento e terapia específica para aprender a ouvir, de acordo com as especificações do Grupo de Implante Coclear do Hospital das Clínicas e Faculdade de Medicina da Universidade de São Paulo (2005), expostas na introdução deste.

É importante ressaltar que apenas um (1) dos estudos analisados relatou que um dos participantes se negou a continuar a terapia após o implante coclear e um relatou não indicar o procedimento a outras pessoas e que não o faria se tivesse que escolher novamente (MURAKAMI et al., 2001).

De acordo com o levantamento realizado pela presente revisão sistemática, os impactos sociais advindos do implante coclear na vida da pessoa implantada são: a) a vergonha relacionada ao dispositivo externo, por ser visível e afirmar a deficiência auditiva; b) dificuldades quanto à fala e compreensão; c) possibilidade de haver rejeição da pessoa implantada por parte do grupo ouvinte pela dificuldade deste em falar; c) possibilidade de desempenhar atividades das quais não participava antes do implante; d) mais autonomia ao interagir com ouvintes; e) autoconfiança e autoestima, possibilitando uma melhor comunicação e participação em atividades sociais, no caso de pessoas com surdez pós-lingual.

\section{Considerações finais}

A partir deste estudo de revisão sistemática foi possível observar que os efeitos sociais na vida da pessoa implantada em decorrência do IC tendem a ser negativos em relação ao aspecto físico do dispositivo externo, podendo resultar em sentimento de inferioridade, vergonha e insegurança. Outro fenômeno observado foi que pessoas que fizeram o implante coclear em decorrência de surdez pós-lingual demonstraram efeitos sociais positivos, tais como ganho de autoconfiança e segurança em situações de comunicação cotidianas e interações sociais.

Ainda foi observado que pode haver rejeição da pessoa implantada por parte do grupo ouvinte, ocasionada pela dificuldade desta em falar. Os efeitos são distintos em 
http://dx.doi.org/10.5902/1984686X38002

adolescentes e adultos provavelmente por toda mudança hormonal, corporal e psicossocial enfrentadas pelos adolescentes nesta etapa de seu desenvolvimento.

Nos estudos analisados se observou que os dados positivos, concernentes ao implante coclear, foram mais valorizados que os negativos. É importante destacar que estes dados auxiliam a aperfeiçoar a prática de reabilitação da pessoa implantada, possibilitando uma melhora significativa na satisfação deste indivíduo. Uma vez que estes dados são desvalorizados ou não têm o tratamento adequado, podem enviesar o estudo de tal forma a levar a entender que a prática do implante apenas apresenta efeitos positivos na vida do indivíduo quando, na realidade, foram encontradas evidências de que existem efeitos sociais negativos decorrentes do IC.

Um dos estudos descreveu a surdez como impedimento de crescimento pessoal e profissional e que a pessoa que não ouve possui sua autoimagem prejudicada e baixa autoestima, assim como experiência sentimento de inferioridade em relação aos ouvintes. Tal achado possibilita o entendimento de que a pessoa surda não se sente bem com ela por não ouvir, no entanto, há relatos na literatura especializada de que o contexto social também pode promover tais sentimentos e não necessariamente o déficit auditivo/surdez.

Foi notado que as queixas quanto ao aspecto do dispositivo, insatisfação com a autoimagem após o implante, vergonha e sentimentos de inferioridade foram relatadas pelos participantes com idade entre 13 e 21 anos, em sua maioria do sexo feminino. Os demais participantes não alegaram insatisfação com a aparência do aparelho, levantando o seguinte questionamento: por quais motivos os participantes do sexo masculino, em idade adulta, não relataram desconfortos e insatisfação referentes ao dispositivo?

O estudo indica que uma revisão sistemática sobre o tema seja realizada em âmbito internacional com a finalidade de analisar a produção científica na área. Sugere, ainda, ser necessário pesquisar mais os efeitos subjetivos do IC em indivíduos implantados com o propósito de compreender como o implante afeta a qualidade de vida dessas pessoas.

Ressalta, por fim, a importância do questionamento sobre o número de estudos publicados que tratam dos efeitos sociais resultantes do implante coclear na vida da pessoa implantada. Este é ainda muito baixo, como mostra o levantamento de dados da presente pesquisa. O que estará ocorrendo com estes indivíduos, em se tratando dos aspectos psicossociais advindos do implante? Qual a relação do implante coclear e a autoimagem desta pessoa? O fato de ter a possibilidade de ouvir melhor corrobora com sua autoimagem, satisfação pessoal e autoestima? 
http://dx.doi.org/10.5902/1984686X38002

Acredita-se que os objetivos tenham sido alcançados a partir do procedimento de estudo realizado com a presente revisão sistemática, contudo vale ressaltar que a pesquisa teve como fator limitante a dificuldade em encontrar um número significativo de fontes científicas dentro dos requisitos de inclusão e exclusão estabelecidos. As autoras esperam que este estudo possa contribuir para a temática e enriquecer o estudo na área dos efeitos sociais decorrentes do implante coclear e que, a partir dele, novas investigações sejam realizadas, buscando sempre estudar e aprimorar o tema.

\section{Referências}

AKOBENG, Anthony Kwaku. Understanding systematic reviews and meta-analysis. Archives of Disease in Childhood, 2005, p. 845 - 848. [Online]. Disponível em: https://www.ncbi.nlm.nih.gov/pmc/articles/PMC1720526/pdf/v090p00845.pdf. Acesso em: 20 jan. 2019.

AMARAL, Vera Lúcia do. Psicologia da Educação. A Psicologia da adolescência - Aula 05. Natal, EDUFRN, 2007, p. 01 - 12. Disponível em:

http://www.ead.uepb.edu.br/arquivos/cursos/Geografia_PAR_UAB/Fasciculos\%20\%20Material/Psicologia_Educacao/Psi_Ed_A05_J_GR_20112007.pdf. Acesso em: 22 jan. 2019.

BUARQUE, Laísa Flávia Soares Fernandes Peixoto et al. Satisfação dos usuários do implante coclear com perda auditiva pós-lingual. Rev. CEFAC, vol. 16 no. 4. São Paulo, 2014, p. 1078 - 1087. Disponível em: http://www.scielo.br/pdf/rcefac/v16n4/19820216-rcefac-16-4-1078.pdf. Acesso em: 20 jan. 2019.

CARVALHO, Circe Nardon. Implante Coclear no Sul do Brasil: Realidade ou Fantasia? Monografia de Conclusão de Curso. Orientadora: Mirian Goldenberg. Centro de Especialização em Fonoaudiologia Clínica Audiologia Clínica. Porto Alegre, 1999, 61 p. Disponível em: http://www.cefac.br/library/teses/16dff7988edd3fb44e1b424efd9afa9f.pdf. Acesso em: 10 jan. 2019.

CORDEIRO, Alexander Magno et al. Grupo de Estudo de Revisão Sistemática do Rio de Janeiro. Revisão sistemática: uma revisão narrativa. Rev. Col. Bras. Cir., vol. 34, n. 6, 2007, p. 428 - 431. [Online]. Disponível em: http://www.scielo.br/pdf/rcbc/v34n6/11.pdf. Acesso em: 09 fev. 2019.

COSTA, Angelo Brandelli; ZOLTOWSKI, Ana Paula Couto. Como escrever um artigo de revisão sistemática. In.: KOLLER, Silvia, COUTO, Maria Clara de Paula, HOHENDORFF, Jean Von (Orgs). Manual de produção científica. Porto Alegre, Artmed, 2014, p. 55 - 70. [Recurso eletrônico].

FERNÁNDEZ-RíOS, Luís; BUELA-CASAL, Gualberto. Standards for the preparation and writing of Psychology review articles. International Journal of Clinical and Health Psychology, vol. 9, no 2, 2009, p. 329 - 344. [Online]. Disponível em: http://www2.uned.es/reop/documentos/standards.pdf. Acesso: 30 de setembro de 2018. 
IMPLANTE Coclear. Grupo de Implante Coclear do Hospital de Clínicas -Faculdade Medicina - USP. São Paulo, 2005, s/p. [Online]. Disponível em: http:// http://www.implantecoclear.org.br/?p=43. Acesso em: 20 fev. 2019.

LICHTIG, Ida et al. O Implante Coclear e a Comunidade Surda: Desafio ou solução? Seminário ATIID - Acessibilidade, TI e Inclusão Digital. Anais eletrônicos... São Paulo, 2003, 05 p. [Online]. Disponível em:

http://www.fsp.usp.br/acessiblidadehttps://www.researchgate.net/publication/267831344_O_ IMPLANTE_COCLEAR_E_A_COMUNIDADE_SURDA_DESAFIO_OU_SOLUCAO. Acesso em: 30 dez. 2018.

MURAKAMI, Gisele Aparecida de Oliveira et al. Expectativas prévias ao implante coclear e avaliação pós-implante em adolescentes. Rev. Estudos de Psicologia, 18 (2). Campinas, 2001, p. 05 - 16. Disponível em: http://www.scielo.br/scielo.php?pid=S0103166X2001000200001\&script=sci_abstract\&tIng=pt. Acesso em: 30 jan. 2019.

NUNES, Leila Regina d'Oliveira de Paula; FERREIRA, Júlio Romero; MENDES, Eniceia Gonçalves. Anexo VI Roteiro de Análise de Teses e Dissertações. In: Análise crítica das teses e dissertações sobre Educação Especial nas áreas de Educação e Psicologia.

PRODICS IV - FAPESP / Relatório Final. São Paulo, FAPESP, 2002, p. 328 - 335.

REZENDE, Patrícia Luiza Ferreira. Implante Coclear na constituição dos sujeitos surdos. [Tese]. Orientadora: Ronice Müller de Quadros; Coorientadora: Maura Corcini Lopes. Florianópolis, 2010, 164 p. Disponível em:

https://repositorio.ufsc.br/handle/123456789/94074. Acesso em: 22 jan. 2019.

SANT'ANNA, Sandra Barreto Giorgi; EICHNER, Andréa Cristina de Oliveira; GUEDES, Mariana Cardoso. Benefício do implante coclear em indivíduos adultos com surdez prélingual. O Mundo da Saúde, abr/jun, 32(2). São Paulo, 2008, p. 238 - 242. Disponível em: https://www.saocamilo-sp.br/pdf/mundo_saude/59/238a242.pdf. Acesso em: 29 jan. 2019.

SACKS, Oliver. Vendo Vozes: uma Jornada pelo Mundo dos Surdos. Rio de Janeiro, Companhia das Letras, 2010, $200 \mathrm{p}$.

SILVA, Josana Carla Gomes Soares; PAULINO, Vanessa Cristina; COSTA, Maria Da Piedade Resende da. Revisão sistemática acerca dos efeitos sociais do implante coclear na vida da pessoa implantada. In: Anais do Congresso Brasileiro de Educação Especial, 2018, São Carlos. Campinas, GALOÁ, 2018. 12 p. [Online]. Disponível em: https://proceedings.science/cbee/cbee-2018/papers/revisao-sistematica-acerca-dos-efeitossociais-do-implante-coclear-na-vida-da-pessoa-implantada. Acesso em: 12 jan. 2019.

SILVA, Paulo Sérgio Modesto da. O Desenvolvimento da Adolescência na Teoria de Piaget. Psicologia. O portal dos Psicólogos, 2011, 06 p. [Online]. Disponível em: http://www.psicologia.pt/artigos/textos/TL0250.pdf. Acesso em: 04 fev. 2019.

TEFILI, Diego et al. Implantes cocleares: aspectos tecnológicos e papel socioeconômico. Rev. Bras. Eng. Bioméd, vol. 29, n. 4, 2013, p. 414 - 433. Disponível em: http://dx.doi.org/10.4322/rbeb.2013.039. Acesso em: 24 jan. 2019. 
TSUKAMOTO, Neide Mitiyo Shimazaki; FIALHO, Neusa Nogueira. A TECNOLOGIA DO IMPLANTE COCLEAR A FAVOR DA EXPRESSÃO BILÍNGUE PARA OS SURDOS. X ANPED SUL, Florianópolis, outubro de 2014. Anais eletrônicos... Florianópolis, 2014, 14 p. Disponível em: http://xanpedsul.faed.udesc.br/arq_pdf/367-0.pdf. Acesso em: 24 jan. 2019.

YAMADA, Midori Otake; BEVILACQUA, Maria Cecília. Dimensão afetiva da pessoa com surdez adquirida, antes e após o implante coclear. Estud. psicol., vol. 29, n. 1. Campinas, 2012, p. 63 - 69. [Online]. Disponível em: http:

//www.scielo.br/pdf/estpsi/v29n1/a07v29n1.pdf. Acesso em: 28 dez. 2018.

ZANARDI, Maria Bozelli; YAMADA, Midori Otake; BEVILACQUA, Maria Cecília. A experiência do adolescente usuário de implante coclear. Psicologia em Revista, v. 15. Belo Horizonte, 2009, p. 69 - 89. Disponível em:

http://pepsic.bvsalud.org/scielo.php?script=sci_arttext\&pid=S1677-11682009000100005. Acesso em: 28 jan. 2019.

\section{Notas}

${ }^{1} \mathrm{Em}$ seu sentido figurado, se refere a um estágio no qual não se está mais fazendo progresso.

${ }^{2}$ Os estudos discriminam a idade, privação auditiva e uso de IC dos participantes relatando a idade da pessoa mais jovem e da pessoa mais velha, no entanto, o estudo T5 apresentou apenas a média de idade e dos demais dados, se distinguindo dos demais.

\section{Correspondência}

Josana Carla Gomes Soares Silva - Rodovia Washington Luiz, s/n, São Carlos, São Paulo, Brasil.

CEP: $13565-905$

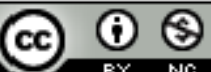

4.0 International (CC BY-NC 4.0) 